\title{
Metrical theorems for inhomogeneous Diophantine approximation in positive characteristic
}

\author{
by \\ MiChAEL FuCHS (Hsinchu) \\ Dedicated to Prof. Harald Niederreiter \\ on the occasion of his 65th birthday
}

1. Introduction. Several recent studies have been concerned with the metric theory of Diophantine approximation in the field of formal Laurent series; for some references see below. The aim of this paper is to make some further progress on the inhomogeneous Diophantine approximation problem. More precisely, we will establish some analogues of results from the real number case (which will be referred to as the "classical case") with some improvements which arise from the more simple nature of the metric structure of the formal Laurent series field.

First, let us fix some notation. We will denote by $\mathbb{F}_{q}$ a finite field with $q$ elements; the polynomial ring, the field of rational functions, and the field of formal Laurent series over $\mathbb{F}_{q}$ will be denoted by $\mathbb{F}_{q}[T], \mathbb{F}_{q}(T)$, and $\mathbb{F}_{q}\left(\left(T^{-1}\right)\right)$, respectively. For $f \in \mathbb{F}_{q}\left(\left(T^{-1}\right)\right)$ with

$$
f=a_{n} T^{n}+a_{n-1} T^{n-1}+\cdots, \quad a_{k} \in \mathbb{F}_{q}, a_{n} \neq 0, n \in \mathbb{Z},
$$

we define $|f|:=q^{n}$ and $|0|:=0$. It is easily checked that $|\cdot|$ is a norm which has the ultra-metric property, i.e.,

$$
|f-g| \leq \max \{|f|,|g|\}
$$

with equality if $|f| \neq|g|$. This property in particular implies that two balls (defined in the standard way) are either disjoint or contained in each other. Finally, we set

$$
\mathbb{L}=\left\{f \in \mathbb{F}_{q}\left(\left(T^{-1}\right)\right):|f|<1\right\} .
$$

2010 Mathematics Subject Classification: 11J61, 11J83, 11K60.

Key words and phrases: formal Laurent series, inhomogeneous Diophantine approximation, Diophantine approximation with restricted denominators, strong laws of large numbers, Schmidt's method. 
Note that $\mathbb{L}$ equipped with the restriction of the norm to $\mathbb{L}$ is a compact abelian group. Consequently, there exists a unique, translation-invariant probability measure which will be denoted by $m$.

In the following, we will be concerned with the inhomogeneous Diophantine approximation problem: for $f, g \in \mathbb{L}$ consider the Diophantine inequality

$$
|Q f-g-P|<\frac{1}{q^{n+l_{n}}}, \quad Q \text { is monic, } \operatorname{deg} Q=n,
$$

whose solutions are pairs of polynomials $\langle P, Q\rangle \in \mathbb{F}_{q}[T] \times \mathbb{F}_{q}[T]$ with $Q \neq 0$ (throughout this work we will use $\langle\cdot, \cdot\rangle$ to denote pairs, whereas $(\cdot, \cdot)$ is reserved for the gcd). Here, $l_{n}$ is a sequence of non-negative integers. In particular, note that $l_{n}$ just depends on $\operatorname{deg} Q$.

In a recent paper, C. Ma and W.-Y. Su [8] investigated the above problem and proved a Khinchin type 0-1 law for the number of solutions if both $f$ and $g$ are chosen randomly (with respect to $m$ ) from $\mathbb{L}$. Their result is an analogue of a result of J. W. S. Cassels [3] from the classical case, where this situation is sometimes called the "double-metric" case. Moreover, the following two "single-metric" cases were considered over the real number field as well (e.g., see [11] and [12]): (S1) fix $f$ and choose a random $g \in \mathbb{L}$; (S2) fix $g$ and choose a random $f \in \mathbb{L}$.

In this paper, we are interested in stochastic properties of the solution set of (1) for $f, g$ such that the number of solutions is infinite. More precisely, we will derive strong laws of large numbers with error terms for the number of solutions $\langle P, Q\rangle$ of (1) with $\operatorname{deg} Q \leq N$. Such results have so far only been established for (S2) with $g=0$; see [6] and H. Nakada and R. Natsui [9]. Here, we will further improve these results and extend them to general $g$. So, the main part of the paper will focus on the case (S2). The other "single-metric" case and the "double-metric" case exhibit a somehow different behavior and will be only briefly discussed in the final section.

From now on, let $g \in \mathbb{L}$ be fixed. Moreover, define

$$
\Psi(N):=\sum_{n \leq N} \frac{1}{q^{l_{n}}} .
$$

Our first result reads as follows.

Theorem 1. The number of solutions of (1) with $0 \leq \operatorname{deg} Q \leq N$ is

$$
\Psi(N)+\mathcal{O}\left(\Psi(N)^{1 / 2}(\log \Psi(N))^{2+\epsilon}\right), \quad \text { a.s. },
$$

where $\epsilon>0$ is an arbitrary constant.

This result is an analogue of a result of W. M. Schmidt [11] from the classical case. In fact, we will use a variant of Schmidt's method to prove it. Note, however, that the error term is better than the one from the classical case. Moreover, no monotonicity assumption on $l_{n}$ is required. 
For $g=0$ the improved error term was also achieved in the classical case; see G. Harman [7]. The result in this special case improves upon Theorem 3 in [9] by removing some further technical conditions on $l_{n}$ and providing an error term. Moreover, our result completes the main result in [4] which was concerned with Diophantine approximation of linear forms with at least two terms. Here, the missing case of only one term is considered. As in the real case, the current situation turns out to be more complex, a claim which is further supported by the fact that the result in [4] has a better error term; for a discussion of this phenomenon in the real case see [10].

In fact, our method of proof can be used to obtain even more general results. More precisely, the method will allow us to investigate inhomogeneous Diophantine approximation with restricted denominators as well. Therefore, replace (1) by

$$
|F(Q) f-g-P|<\frac{1}{q^{n+l_{n}}}, \quad Q \text { is monic, } \operatorname{deg} Q=n,
$$

where $l_{n}$ is as above and $F$ is a function from $\mathbb{F}_{q}[T]$ into $\mathbb{F}_{q}[T]$.

First, we will fix some further notation. Let

$$
\mathcal{F}:=\{Q: Q \text { monic and } F(Q) \neq 0\}
$$

and denote by $\mathcal{F}_{n}$ the subset of all polynomials $Q \in \mathcal{F}$ with $\operatorname{deg} Q=n$. Subsequently, we will only consider $F$ with the following property: for $Q, Q^{\prime} \in \mathcal{F}$ with $\operatorname{deg} Q \leq \operatorname{deg} Q^{\prime}$, we have $\operatorname{deg} F(Q) \leq \operatorname{deg} F\left(Q^{\prime}\right)$. Finally, set

$$
\Psi(N, \mathcal{F}):=\sum_{n \leq N} \frac{\# \mathcal{F}_{n}}{q^{n+l_{n}}}
$$

Then the following generalization of the above result holds.

Theorem 2. Assume that $F(Q)$ is either $Q$ or 0 . Then the number of solutions of (2) with $Q \in \mathcal{F}$ and $0 \leq \operatorname{deg} Q \leq N$ is

$$
\Psi(N, \mathcal{F})+\mathcal{O}\left(\Psi(N)^{1 / 2}(\log \Psi(N))^{2+\epsilon}\right), \quad \text { a.s. },
$$

where $\epsilon>0$ is an arbitrary constant.

In particular, the latter result gives a meaningful asymptotic formula whenever

$$
\liminf _{n \rightarrow \infty} \frac{\# \mathcal{F}_{n}}{q^{n}}>0 .
$$

Two important special cases are collected in the following corollary, the first of which has to be compared with the results in [6].

Corollary 1.

(i) Let $C, D \in \mathbb{F}_{q}[T]$ with $\operatorname{deg} C<\operatorname{deg} D$. Then the number of solutions of (1) with $Q \equiv C \bmod D$ and $0 \leq \operatorname{deg} Q \leq N$ is 


$$
\frac{1}{|D|} \Psi(N)+\mathcal{O}\left(\Psi(N)^{1 / 2}(\log \Psi(N))^{2+\epsilon}\right), \quad \text { a.s. },
$$

where $\epsilon>0$ is an arbitrary constant.

(ii) The number of solutions of (1) with $Q$ monic, square-free and $0 \leq$ $\operatorname{deg} Q \leq N$ is

$$
\frac{q-1}{q} \Psi(N)+\mathcal{O}\left(\Psi(N)^{1 / 2}(\log \Psi(N))^{2+\epsilon}\right), \quad \text { a.s. },
$$

where $\epsilon>0$ is an arbitrary constant.

Note that condition (4) is not satisfied for some interesting $\mathcal{F}$ such as the set of monic, irreducible polynomials. This situation, however, turns out to be simpler and we can obtain a strong law of large numbers with an even better error term. Therefore, we first prove an analogue of Theorem 3.1 in [7] which holds for general $F$.

THEOREM 3. The number of solutions of (2) with $Q \in \mathcal{F}$ and $0 \leq$ $\operatorname{deg} Q \leq N$ is

$$
\Psi(N, \mathcal{F})+\mathcal{O}\left(\Psi_{0}(N)^{1 / 2}\left(\log \Psi_{0}(N)\right)^{3 / 2+\epsilon}\right), \quad \text { a.s. },
$$

where $\epsilon>0$ is an arbitrary constant and

$$
\Psi_{0}(N)=\sum_{n \leq N} \frac{1}{q^{n+l_{n}}} \sum_{m \leq n} \sum_{Q \in \mathcal{F}_{n}} \sum_{Q^{\prime} \in \mathcal{F}_{m}} \frac{\left|\left(F(Q), F\left(Q^{\prime}\right)\right)\right|}{|F(Q)|} .
$$

This result entails the following corollary.

Corollary 2.

(i) Let

$$
\Psi_{1}(N):=\sum_{n \leq N} \frac{1}{n q^{l_{n}}} .
$$

Then the number of solutions of (1) with $Q$ monic, irreducible and $0 \leq \operatorname{deg} Q \leq N$ is

$$
\Psi_{1}(N)+\mathcal{O}\left(\Psi_{1}(N)^{1 / 2}\left(\log \Psi_{1}(N)\right)^{3 / 2+\epsilon}\right), \quad \text { a.s. },
$$

where $\epsilon>0$ is an arbitrary constant.

(ii) Let $F(Q)=Q^{t}$ with $t \geq 2$. Then the number of solutions of (2) with $0 \leq \operatorname{deg} Q \leq N$ is

$$
\Psi(N)+\mathcal{O}\left(\Psi(N)^{1 / 2}(\log \Psi(N))^{3 / 2+\epsilon}\right), \quad \text { a.s. },
$$

where $\epsilon>0$ is an arbitrary constant.

It is worth mentioning that Theorem 3 does not give a meaningful result in the situations discussed in Theorem 1 and Corollary 1. Consequently, part (ii) of Corollary 2 shows that the complexities of $t=1$ and $t \geq 2$ are rather different. 
We conclude the introduction by giving a short plan of the paper. In the next section, we will prove a weak independence result which will form the crucial step in deriving all results above. In particular, Theorem 3 will follow rather quickly from this result and this will be demonstrated in the next section as well. Then, in Section 3, we will show how to adapt Schmidt's method to the current situation to obtain a proof of Theorem 11 and Theorem 2 . In the final section, we will then briefly discuss the other "single-metric" case and the "double-metric" case.

Notation. All logarithms appearing throughout this work will only attain values $\geq 1$, i.e., $\log _{a} x$ should be interpreted as $\max \left\{\log _{a} x, 1\right\}$. We will use Landau's notation $f(x)=\mathcal{O}(g(x))$ as well as Vinogradov's notation $f(x) \ll g(x)$ to indicate that there exists a constant $C \geq 0$ such that $|f(x)| \leq C|g(x)|$ for all $x$ sufficiently large.

2. A weak independence result with applications. We start by proving a technical lemma that constitutes a refinement of Lemma 2.3 in [2].

Lemma 1. Let $Q, Q^{\prime}$ be two non-zero polynomials with $n=\operatorname{deg} Q, m=$ $\operatorname{deg} Q^{\prime}$ and $d=\operatorname{deg}\left(Q, Q^{\prime}\right)$. Let l be a non-negative integer. Then the number $N$ of pairs $\left\langle P, P^{\prime}\right\rangle$ with $\operatorname{deg} P<n, \operatorname{deg} P^{\prime}<m$ and

$$
\left|\frac{g+P}{Q}-\frac{g+P^{\prime}}{Q^{\prime}}\right|<\frac{1}{q^{m+l}}
$$

satisfies

$$
N \begin{cases}=q^{n-l} & \text { if } n \geq l+d, \\ \leq q^{d} & \text { if } n<l+d .\end{cases}
$$

Proof. First, (7) can be reformulated to

$$
\left|g\left(Q^{\prime}-Q\right)+P Q^{\prime}-P^{\prime} Q\right|<q^{n-l} .
$$

Next, write $Q=\left(Q, Q^{\prime}\right) \cdot \bar{Q}$ and $Q^{\prime}=\left(Q, Q^{\prime}\right) \cdot \bar{Q}^{\prime}$. Then

$$
\left|g\left(\bar{Q}^{\prime}-\bar{Q}\right)+P \bar{Q}^{\prime}-P^{\prime} \bar{Q}\right|<q^{n-l-d} \text {. }
$$

Let $-C$ denote the polynomial part of $g\left(\bar{Q}^{\prime}-\bar{Q}\right)$. Now, we will consider two cases.

First, assume that $n<l+d$. Then a necessary condition for $\left\langle P, P^{\prime}\right\rangle$ to be a solution of the above inequality is $P \bar{Q}^{\prime}-P^{\prime} \bar{Q}=C$. Observe that for $P$ with $\operatorname{deg} P<n$ and

$$
P \bar{Q}^{\prime} \equiv C \bmod \bar{Q},
$$

we have $P \bar{Q}^{\prime}=C+P^{\prime} \bar{Q}$ with some polynomial $P^{\prime}$ and

$$
\operatorname{deg} P^{\prime}+\operatorname{deg} \bar{Q}=\operatorname{deg}\left(P \bar{Q}^{\prime}-C\right) \leq \operatorname{deg} P+\operatorname{deg} \bar{Q}^{\prime}<n+\operatorname{deg} \bar{Q}^{\prime} .
$$

Consequently, $\operatorname{deg} P^{\prime}<m$. So, either $N=0$ or $N$ equals the number of solutions of (8) which is $q^{d}$. 
Next, we consider $n \geq l+d$. Here, we can argue similarly, the only difference being that $N$ equals the number of solutions of (8) with $C$ replaced by $C+D$ for all polynomials $D$ with $\operatorname{deg} D<n-l-d$. Consequently, $N=q^{n-l}$.

Next, for $Q \in \mathcal{F}_{n}$ we define

$$
F_{Q}:=\left\{f \in \mathbb{L}: f \text { satisfies }(2) \text { with some } P \in \mathbb{F}_{q}[T]\right\} .
$$

Obviously, $F_{Q}$ is the union of $|F(Q)|$ disjoint balls. Consequently,

$$
m\left(F_{Q}\right)=\frac{1}{q^{n+l_{n}}} .
$$

Moreover, we have the following weak independence result. Then

Proposition 1. Let $Q \in \mathcal{F}_{n}, Q^{\prime} \in \mathcal{F}_{m}$, and $d=\operatorname{deg}\left(F(Q), F\left(Q^{\prime}\right)\right)$.

$$
m\left(F_{Q} \cap F_{Q^{\prime}}\right) \leq m\left(F_{Q}\right) m\left(F_{Q^{\prime}}\right)+q^{d-\operatorname{deg} F(Q)-n-l_{n}} .
$$

Proof. First assume that $n+l_{n}+\operatorname{deg} F(Q) \geq m+l_{m}+\operatorname{deg} F\left(Q^{\prime}\right)$. Then all balls which make up $F_{Q}$ have radius at most as large as the radius of the balls which make up $F_{Q^{\prime}}$. So, by the ultra-metric property of the norm, we have to count how many of the balls with center $(g+P) / F(Q)$ are contained in balls with center $\left(g+P^{\prime}\right) / F\left(Q^{\prime}\right)$ and radius $q^{-\operatorname{deg} F\left(Q^{\prime}\right)-m-l_{m}}$, i.e., we have to count the number of solutions of

$$
\left|\frac{g+P}{F(Q)}-\frac{g+P^{\prime}}{F\left(Q^{\prime}\right)}\right|<\frac{1}{q^{\operatorname{deg} F\left(Q^{\prime}\right)+m+l_{m}}} .
$$

The latter number is given by the above lemma. We first consider the case with $\operatorname{deg} F(Q) \geq m+l_{m}+d$. Here, the number of solutions equals $q^{\operatorname{deg} F(Q)-m-l_{m}}$. So, we obtain

$$
m\left(F_{Q} \cap F_{Q^{\prime}}\right)=\frac{|F(Q)| q^{-m-l_{m}}}{|F(Q)| q^{n+l_{n}}}=\frac{1}{q^{n+l_{n}}} \cdot \frac{1}{q^{m+l_{m}}}=m\left(F_{Q}\right) m\left(F_{Q^{\prime}}\right) .
$$

Hence, the assertion holds in this case. Now, consider the second case where $\operatorname{deg} F(Q)<m+l_{m}+d$. Then, again by the above lemma,

$$
m\left(F_{Q} \cap F_{Q^{\prime}}\right) \leq \frac{q^{d}}{q^{\operatorname{deg} F(Q)+n+l_{n}}} .
$$

Hence, the claim is proved in this case as well.

Next, if $n+l_{n} \operatorname{deg} F(Q)<m+l_{m}+\operatorname{deg} F\left(Q^{\prime}\right)$, we obtain from the arguments above the claim with the second term replaced by $q^{d-\operatorname{deg} F\left(Q^{\prime}\right)-m-l_{m}}$. This term is trivially bounded by $q^{d-\operatorname{deg} F(Q)-n-l_{n}}$. Hence, the proof of the proposition is finished.

The above proposition will turn out to be one of the key ingredients in the proof of our results. Another key ingredient is the following important lemma which is a standard tool in metric number theory. 
Lemma 2 (Lemma 1.5 in [7]). Let $\xi_{n}(\omega)$ be a sequence of non-negative random variables defined on a probability space $(\Omega, \mathcal{B}, P)$. Let $\psi_{n}$ and $\varphi_{n}$ be sequences of real numbers with $0 \leq \psi_{n} \leq \varphi_{n}$. Define

$$
\Phi(N)=\sum_{n \leq N} \varphi_{n}
$$

and assume that $\Phi(N) \rightarrow \infty$ as $N \rightarrow \infty$. Finally, assume that

$$
\mathbb{E}\left(\sum_{M \leq n \leq N} \xi_{n}-\psi_{n}\right)^{2} \ll \sum_{M \leq n \leq N} \varphi_{n}
$$

for all non-negative $M<N$. Then

$$
\sum_{n \leq N} \xi_{n}(\omega)=\sum_{n \leq N} \psi_{n}+\mathcal{O}\left(\Phi(N)^{1 / 2}(\log \Phi(N))^{3 / 2+\epsilon}+\max _{n \leq N} \psi_{n}\right), \quad \text { a.s. },
$$

where $\epsilon>0$ is an arbitrary constant.

As a first application of this lemma, we show how to deduce Theorem 3 from it. Therefore, set

$$
\xi_{n}:=\#\{\langle P, Q\rangle:\langle P, Q\rangle \text { is a solution of (2) }\} .
$$

This sequence of random variables has the following properties.

Proposition 2.

$$
\mathbb{E}\left(\sum_{n \leq N} \xi_{n}\right)=\Psi(N, \mathcal{F})
$$

$$
\mathbb{E}\left(\sum_{M \leq n \leq N} \xi_{n}-\frac{\# \mathcal{F}_{n}}{q^{n+l_{n}}}\right)^{2} \ll \sum_{M \leq n \leq N} \frac{1}{q^{n+l_{n}}} \sum_{m \leq n} \sum_{Q \in \mathcal{F}_{n}} \sum_{Q^{\prime} \in \mathcal{F}_{m}} \frac{\left|\left(F(Q), F\left(Q^{\prime}\right)\right)\right|}{|F(Q)|}
$$

for all non-negative integers $M<N$.

Proof. Part (i) follows from

$$
\xi_{n}=\sum_{Q \in \mathcal{F}_{n}} \mathbf{1}_{F_{Q}}
$$

and basic properties of the mean value.

For (ii), we also use the above representation which yields

$$
\begin{aligned}
& \mathbb{E}\left(\sum_{M \leq n \leq N} \xi_{n}-\frac{\# \mathcal{F}_{n}}{q^{n+l_{n}}}\right)^{2} \\
& =2 \sum_{M \leq n \leq N} \sum_{M \leq m \leq n-1} \sum_{Q \in \mathcal{F}_{n}, Q^{\prime} \in \mathcal{F}_{m}}\left(m\left(F_{Q} \cap F_{Q^{\prime}}\right)-m\left(F_{Q}\right) m\left(F_{Q^{\prime}}\right)\right) \\
& \quad+\sum_{M \leq n \leq N} \sum_{Q \in \mathcal{F}_{n}, Q^{\prime} \in \mathcal{F}_{m}}\left(m\left(F_{Q} \cap F_{Q^{\prime}}\right)-m\left(F_{Q}\right) m\left(F_{Q^{\prime}}\right)\right) .
\end{aligned}
$$

Applying Proposition 1 immediately yields the claimed result. 
Now, we can prove Theorem 3 .

Proof of Theorem 3. If $\Psi(N, \mathcal{F}) \rightarrow c \geq 0$ as $N \rightarrow \infty$, the result follows by a standard application of the Borel-Cantelli lemma. Hence, we can assume that $\Psi(N, \mathcal{F}) \rightarrow \infty$ as $N \rightarrow \infty$. But then the claim follows from the proposition above together with Lemma 2 .

Corollary 2 follows from the last result as follows.

Proof of Corollary 2. For (i), we use the well-known result (see Chapter 3 in [1])

$$
\# \mathcal{F}_{n}=q^{n} / n+\mathcal{O}\left(q^{\epsilon n}\right),
$$

where $\epsilon<1$ is a suitable constant. Hence,

$$
\Psi(N, \mathcal{F})=\Psi_{1}(N)+\mathcal{O}(1) .
$$

Moreover,

$$
\begin{aligned}
\Psi_{0}(N) & =\sum_{n \leq N} \frac{1}{q^{2 n+l_{n}}} \sum_{m \leq n} \sum_{\begin{array}{c}
\operatorname{deg} Q=n \\
Q \text { monic, irreducible }
\end{array}} \sum_{\begin{array}{c}
\operatorname{deg} Q^{\prime}=m \\
Q^{\prime} \text { monic, irreducible }
\end{array}}\left|\left(Q, Q^{\prime}\right)\right| \\
& \ll \Psi_{1}(N),
\end{aligned}
$$

where the last estimate again follows by (9). This proves the claim.

As for (ii), first observe that $\# \mathcal{F}_{n}=q^{n}$ and hence $\Psi(N, \mathcal{F})=\Psi(N)$. The bound for $\Psi_{0}(N)$ is slightly more tricky. First,

$$
\begin{aligned}
& \Psi_{0}(N)=\sum_{n \leq N} \frac{1}{q^{(t+1) n+l_{n}}} \sum_{m \leq n} \sum_{\substack{\operatorname{deg} Q=n \\
Q \text { monic }}} \sum_{\substack{\operatorname{deg} Q^{\prime}=m \\
Q^{\prime} \text { monic }}}\left|\left(Q^{t},\left(Q^{\prime}\right)^{t}\right)\right| \\
& \ll \sum_{n \leq N} \frac{1}{q^{(t+1) n+l_{n}}} \sum_{\substack{\operatorname{deg} Q=n \\
Q \text { monic }}} \sum_{\substack{D \mid Q \\
D \text { monic }}} \frac{q^{n}}{|D|}|D|^{t} .
\end{aligned}
$$

Next, we have

$$
\sum_{\substack{\operatorname{deg} Q=n \\ Q \text { monic }}} \sum_{\substack{D \mid Q \\ D \text { monic }}}|D|^{t-1}=\sum_{\substack{d \leq n \\ \operatorname{deg} D=d \\ D \text { monic }}} \sum_{|D|} \frac{q^{n}}{|D|^{t-1}}=q^{n} \sum_{d \leq n} q^{(t-1) d} \ll q^{t n} .
$$

Plugging this into the estimate above yields $\Psi_{0}(N) \ll \Psi(N)$. Hence, the result is established.

3. Schmidt's method in positive characteristic. Note that the method from the last section does not yield a meaningful result for the case $F(Q)=Q$. More specifically, it is easily checked that the error term from the proof of Corollary 2(ii) for $t=1$ would be larger than the main term. The same phenomenon also occurs in the real case, where this problem 
was overcome by an ingenious method introduced by W. M. Schmidt in 10 and [11. In this section, Schmidt's method will be adapted to the current situation.

We start with a couple of (easy) lemmas.

Lemma 3 (Dirichlet's principle in positive characteristic). For all nonzero polynomials $Q$ there exist polynomials $A, B$ with $0<|A| \leq|Q|$ and $(A, B)=1$ such that

$$
\left|g-\frac{B}{A}\right|<\frac{1}{|A||Q|}
$$

Proof. This is proved as in the classical case.

Observe that $A$ and $B$ in the previous lemma just depend on $\operatorname{deg} Q$. Subsequently, for any given non-zero polynomial $Q$, we will choose a fixed pair $\langle A, B\rangle$ satisfying the assumption of the previous lemma for a polynomial $Q^{\prime}$ with $\operatorname{deg} Q^{\prime}=\lfloor\operatorname{deg} Q / 2\rfloor$.

Next, we define the following two sets:

$$
\begin{aligned}
S(Q ; k) & =\{P: \operatorname{deg} P<\operatorname{deg} Q \text { and } \operatorname{deg}(P, Q) \leq k\}, \\
S^{*}(Q ; k) & =\{P: \operatorname{deg} P<\operatorname{deg} Q \text { and } \operatorname{deg}(A P+B, Q) \leq k\},
\end{aligned}
$$

whose cardinalities will be denoted by $\varphi(Q ; k)$ and $\varphi^{*}(Q ; k)$, respectively.

Lemma 4. We have

$$
\varphi^{*}(Q ; k) \geq \varphi(Q ; k) .
$$

Proof. First, let $Q=Q_{1} Q_{2}$, where every prime factor of $Q_{1}$ is also a prime factor of $A$ and $\left(Q_{2}, A\right)=1$. Then

$$
\varphi(Q ; k) \leq \varphi\left(Q_{1} ; k\right) \varphi\left(Q_{2} ; k\right) \leq\left|Q_{1}\right| \varphi\left(Q_{2} ; k\right) .
$$

Now, note that $A P+B$ with $\operatorname{deg} P<\operatorname{deg} Q_{2}$ are all different modulo $Q_{2}$. Hence, $\varphi\left(Q_{2} ; k\right)=\#\left\{P: \operatorname{deg} P<\operatorname{deg} Q_{2}\right.$ and $\left.\operatorname{deg}\left(A P+B, Q_{2}\right) \leq k\right\}$. Finally, notice that

$$
\left(A P+B, Q_{2}\right)=\left(A P+B, Q_{1} Q_{2}\right)=(A P+B, Q) .
$$

Consequently,

$$
\varphi^{*}(Q ; k)=\left|Q_{1}\right| \cdot \#\left\{P: \operatorname{deg} P<\operatorname{deg} Q_{2} \text { and } \operatorname{deg}\left(A P+B, Q_{2}\right) \leq k\right\} .
$$

Combining everything yields the claimed result.

Next, we fix $F(Q)=Q$. Moreover, as in the last section, it suffices to consider the case where $\Psi(N) \rightarrow \infty$ as $N \rightarrow \infty$. The method of the last section does not work when directly applied to the sequence $\xi_{n}$. Therefore, we will approximate this sequence by

$$
\xi_{n}^{*}:=\#\left\{\langle P, Q\rangle: P \in S^{*}(Q ; \Gamma(n)) \text { and }\langle P, Q\rangle \text { is a solution of (1) }\right\},
$$


where $\Gamma(n)=\left\lfloor\log _{q} \Psi(n)^{2}\right\rfloor$. Moreover, as in the last section, we define $F_{Q}^{*}:=\left\{f \in \mathbb{L}: f\right.$ satisfies (1) with some $\left.P \in S^{*}(Q ; \Gamma(n))\right\}$.

Then

$$
\xi_{n}^{*}=\sum_{\substack{\operatorname{deg} Q=n \\ Q \text { monic }}} \mathbf{1}_{F_{Q}^{*}}
$$

and consequently

$$
\mathbb{E} \xi_{n}^{*}=\sum_{\substack{\operatorname{deg} Q=n \\ Q \text { monic }}} \frac{\varphi^{*}(Q ; \Gamma(n))}{q^{2 n+l_{n}}} .
$$

The next result shows that the mean values of the partial sums of $\xi_{n}$ and $\xi_{n}^{*}$ are very close to each other.

Proposition 3. We have

$$
\mathbb{E}\left(\sum_{M \leq n \leq N} \xi_{n}^{*}\right)=\sum_{M \leq n \leq N} \frac{1}{q^{l_{n}}}+\mathcal{O}(1)
$$

for all non-negative integers $M<N$.

Proof. First, observe that

$$
\begin{aligned}
0 \leq \sum_{M \leq n \leq N} \frac{1}{q^{l_{n}}}-\mathbb{E}\left(\sum_{M \leq n \leq N} \xi_{n}^{*}\right) & =\sum_{M \leq n \leq N} \sum_{\substack{\operatorname{deg} Q=n \\
Q \text { monic }}} \frac{q^{n}-\varphi^{*}(Q ; \Gamma(n))}{q^{2 n+l_{n}}} \\
& \leq \sum_{M \leq n \leq N} \sum_{\substack{\operatorname{deg} Q=n \\
Q \text { monic }}} \frac{q^{n}-\varphi(Q ; \Gamma(n))}{q^{2 n+l_{n}}},
\end{aligned}
$$

where we have used the above lemma in the last step. Next, it is well-known (see [5]) that the number of pairs $\langle P, Q\rangle$ with $\operatorname{deg} P=l<\operatorname{deg} Q=n, P, Q$ monic and $\operatorname{deg}(P, Q)=k<l$ is given by

$$
q^{n+l-k}\left(1-\frac{1}{q}\right)
$$

Consequently,

$$
\begin{aligned}
\sum_{\substack{\operatorname{deg} Q=n \\
Q \text { monic }}} \varphi(Q, \Gamma(n)) & =\frac{(q-1)^{2}}{q} \sum_{l=\Gamma(n)+1}^{n-1} \sum_{k=0}^{\Gamma(n)} q^{n+l-k}+\mathcal{O}\left(\sum_{l=0}^{\Gamma(n)} \sum_{k=0}^{l} q^{n+l-k}\right) \\
& =q^{2 n}+\mathcal{O}\left(q^{2 n-\Gamma(n)}\right) .
\end{aligned}
$$

Plugging this into the above expression, we obtain

$$
0 \leq \sum_{M \leq n \leq N} \frac{1}{q^{l_{n}}}-\mathbb{E}\left(\sum_{M \leq n \leq N} \xi_{n}^{*}\right) \ll \sum_{N \leq n \leq M} \frac{1}{q^{l_{n}} \Psi(n)^{2}} .
$$


Since the latter series is convergent by the Abel-Dini theorem, the claim is proved.

Finally, we need the following property.

Proposition 4. We have

$$
\mathbb{E}\left(\sum_{M \leq n \leq N} \xi_{n}^{*}-\frac{1}{q^{l_{n}}}\right)^{2} \ll \sum_{M \leq n \leq N} \frac{\Gamma(n)}{q^{l_{n}}}
$$

for all non-negative integers $M<N$.

Proof. We start with an observation that is needed below. By a close inspection of the proof of Proposition 1, we have

$$
m\left(F_{Q}^{*} \cap F_{Q^{\prime}}^{*}\right) \leq \frac{1}{q^{n+l_{n}}} \cdot \frac{1}{q^{m+l_{m}}}+\frac{1}{q^{2 n+l_{n}}} A\left(Q, Q^{\prime}\right),
$$

where $A\left(Q, Q^{\prime}\right)$ is the number of all pairs $P, P^{\prime}$ with $P \in S^{*}(Q ; \Gamma(n)), P^{\prime} \in$ $S^{*}\left(Q^{\prime} ; \Gamma(m)\right)$ and

$$
\left|g\left(Q-Q^{\prime}\right)+P^{\prime} Q-P Q^{\prime}\right|<\min \left\{\left|\left(Q, Q^{\prime}\right)\right|, q^{\max \left\{n-m-l_{m}, m-n-l_{n}\right\}}\right\} .
$$

Moreover, observe that $A\left(Q, Q^{\prime}\right) \leq\left|\left(Q, Q^{\prime}\right)\right|$.

We will use this to bound the expected value from the claim. First,

$$
\begin{aligned}
\mathbb{E}( & \left.\sum_{M \leq n \leq N} \xi_{n}^{*}-\frac{1}{q^{l_{n}}}\right)^{2} \\
= & \sum_{M \leq n \leq N} \sum_{M \leq m \leq N} \mathbb{E} \xi_{n}^{*} \cdot \xi_{m}^{*}-2 \sum_{M \leq n \leq N} \frac{1}{q^{l_{n}}} \mathbb{E}\left(\sum_{N \leq n \leq M} \xi_{n}^{*}\right) \\
& +\sum_{M \leq n \leq N} \sum_{M \leq m \leq N} \frac{1}{q^{l_{n}}} \cdot \frac{1}{q^{l_{m}}} \\
= & \sum_{M \leq n \leq N} \sum_{M \leq m \leq N}\left(\mathbb{E} \xi_{n}^{*} \cdot \xi_{m}^{*}-\frac{1}{q^{l_{n}}} \cdot \frac{1}{q^{l_{m}}}\right)+\mathcal{O}\left(\sum_{M \leq n \leq N} \frac{1}{q^{l_{n}}}\right) \\
= & 2 \sum_{M \leq n \leq N} \sum_{M \leq m \leq n-1}\left(\mathbb{E} \xi_{n}^{*} \cdot \xi_{m}^{*}-\frac{1}{q^{l_{n}}} \cdot \frac{1}{q^{l_{m}}}\right)+\sum_{M \leq n \leq N}\left(\mathbb{E}\left(\xi_{n}^{*}\right)^{2}-\frac{1}{q^{2 l_{n}}}\right) \\
& +\mathcal{O}\left(\sum_{M \leq n \leq N} \frac{1}{q^{l_{n}}}\right),
\end{aligned}
$$

where the third step follows from Proposition 3. Now, applying (10) gives 


$$
\begin{aligned}
\sum_{M \leq m \leq n} \mathbb{E} \xi_{n}^{*} \cdot \xi_{m}^{*} & =\sum_{M \leq m \leq n} \sum_{\substack{\operatorname{deg} Q=n \\
Q \text { monic }}} \sum_{\substack{\operatorname{deg} Q^{\prime}=m \\
Q^{\prime} \text { monic }}} m\left(F_{Q}^{*} \cap F_{Q^{\prime}}^{*}\right) \\
& \leq \frac{1}{q^{l_{n}}} \cdot \sum_{M \leq m \leq n} \frac{1}{q^{l_{m}}}+\frac{1}{q^{2 n+l_{n}}} \sum_{\substack{M \leq m \leq n \operatorname{deg} Q=n \operatorname{deg} Q^{\prime}=m \\
Q \text { monic } \\
Q^{\prime} \text { monic }}} A\left(Q, Q^{\prime}\right) .
\end{aligned}
$$

Using this to bound the first and second term in the expression above yields

$$
\begin{aligned}
& \mathbb{E}\left(\sum_{M \leq n \leq N} \xi_{n}^{*}-\frac{1}{q^{l_{n}}}\right)^{2} \\
& \ll \sum_{M \leq n \leq N} \frac{1}{q^{2 n+l_{n}}} \sum_{M \leq m \leq n} \sum_{\substack{\operatorname{deg} Q=n \operatorname{deg} Q^{\prime}=m \\
Q \text { monic }}} \sum_{Q^{\prime} \text { monic }} A\left(Q, Q^{\prime}\right)+\sum_{M \leq n \leq N} \frac{1}{q^{l_{n}}} .
\end{aligned}
$$

Next, we will estimate

$$
\Sigma:=\sum_{M \leq n \leq N} \frac{1}{q^{2 n+l_{n}}} \sum_{M \leq m \leq n} \sum_{\substack{\operatorname{deg} Q=n \operatorname{deg} Q^{\prime}=m \\ Q \text { monic } \\ Q^{\prime} \text { monic }}} A\left(Q, Q^{\prime}\right) .
$$

Therefore, we fix an arbitrary small $\delta$ and break $\Sigma$ into two parts $\Sigma^{\prime}$ and $\Sigma^{\prime \prime}$, where the first part runs over all pairs $\left\langle Q, Q^{\prime}\right\rangle$ with $\operatorname{deg} Q^{\prime} \leq\lceil n-$ $\left.\delta \operatorname{deg}\left(Q, Q^{\prime}\right)\right\rceil$ and the second part runs over the remaining pairs. In order to bound $\Sigma^{\prime}$, we change the order of summation as follows: first we sum over $Q$, then over $D \mid Q$ and finally over $Q^{\prime}$ with $D=\left(Q, Q^{\prime}\right)$. Note that for fixed $Q$ and $D$ the number of $Q^{\prime \prime}$ s is bounded by $q^{n} /|D|^{1+\delta}$. This together with $A\left(Q, Q^{\prime}\right) \leq|D|$ then yields

$$
\begin{aligned}
\Sigma^{\prime} & =\sum_{M \leq n \leq N} \frac{1}{q^{2 n+l_{n}}} \sum_{\substack{\operatorname{deg} Q=n \\
Q \text { monic }}} \sum_{\substack{D \mid Q \\
D \text { monic }}} \frac{q^{n}}{|D|^{1+\delta}}|D| \\
& \ll \sum_{M \leq n \leq N} \frac{1}{q^{l_{n}}} \sum_{\substack{\operatorname{deg} D \leq n \\
D \text { monic }}} \frac{1}{|D|^{1+\delta}} \ll \sum_{M \leq n \leq N} \frac{1}{q^{l_{n}}} .
\end{aligned}
$$

As for $\Sigma^{\prime \prime}$, observe that $\operatorname{deg} Q^{\prime}>\left\lceil n-\delta \operatorname{deg}\left(Q, Q^{\prime}\right)\right\rceil$ implies

$$
\min \left\{\left|\left(Q, Q^{\prime}\right)\right|, q^{\max \left\{n-m-l_{m}, m-n-l_{n}\right\}}\right\}<\left|\left(Q, Q^{\prime}\right)\right|^{\delta} .
$$

Hence, for all $\left\langle Q, Q^{\prime}\right\rangle$ involved in the range of $\Sigma^{\prime \prime}$ the relation (11) can be replaced by

$$
\left|g\left(Q-Q^{\prime}\right)+P^{\prime} Q-P Q^{\prime}\right|<\left|\left(Q, Q^{\prime}\right)\right|^{\delta} .
$$


This yields

$$
\Sigma^{\prime \prime} \ll \sum_{M \leq n \leq N} \frac{1}{q^{2 n+l_{n}}} \sum_{M \leq m \leq n} \sum_{\substack{\operatorname{deg} Q=n \operatorname{deg} Q^{\prime}=m \\ Q \text { monic } \\ Q^{\prime} \text { monic }}} B\left(Q, Q^{\prime}\right),
$$

where $B\left(Q, Q^{\prime}\right)$ denotes the number of all $P, P^{\prime}$ with $P \in S^{*}(Q ; \Gamma(n))$ and $P^{\prime} \in S^{*}\left(Q^{\prime} ; \Gamma(m)\right)$ that satisfy 13$)$. Again note that $B\left(Q, Q^{\prime}\right) \leq\left|\left(Q, Q^{\prime}\right)\right|$.

Collecting all bounds so far, we see that the right hand side of $(12)$ can be replaced by

$$
\sum_{M \leq n \leq N} \frac{1}{q^{2 n+l_{n}}} \sum_{M \leq m \leq n} \sum_{\substack{\operatorname{deg} Q=n \\ Q \text { monic }}} \sum_{\substack{\operatorname{deg} Q^{\prime}=m \\ Q^{\prime} \text { monic }}} B\left(Q, Q^{\prime}\right)+\sum_{M \leq n \leq N} \frac{1}{q^{l_{n}}} .
$$

Next, we will estimate the first term

$$
\Sigma_{0}:=\sum_{M \leq n \leq N} \frac{1}{q^{2 n+l_{n}}} \sum_{M \leq m \leq n} \sum_{\substack{\operatorname{deg} Q=n \\ Q \text { monic }}} \sum_{\substack{\operatorname{deg} Q^{\prime}=m \\ Q^{\prime} \text { monic }}} B\left(Q, Q^{\prime}\right),
$$

which we will break into three parts $\Sigma_{0}^{\prime}, \Sigma_{0}^{\prime \prime}, \Sigma_{0}^{\prime \prime \prime}$, where the ranges will be given below. For every part we will proceed as for $\Sigma^{\prime}$ above. More precisely, we will change the order of summation as follows: just as for $\Sigma^{\prime}$, the first two sums will run over $Q$ and $D \mid Q$. The final sum will run over $\bar{Q}^{\prime}$ with $\left(\bar{Q}^{\prime}, Q / D\right)=1$. Here, we introduce the notation $Q^{\prime}=D \bar{Q}^{\prime}$ and $Q=D \bar{Q}$. Using this notation, we can rewrite $(13)$ as

$$
\left|g\left(\bar{Q}-\bar{Q}^{\prime}\right)+P^{\prime} \bar{Q}-P \bar{Q}^{\prime}\right|<|D|^{-1+\delta} .
$$

Finally, we need the notation $R=g-B / A$, where $\langle A, B\rangle$ is the pair belonging to $Q$. Now, we will separately estimate the three parts $\Sigma_{0}^{\prime}, \Sigma_{0}^{\prime \prime}, \Sigma_{0}^{\prime \prime \prime}$.

As for $\Sigma_{0}^{\prime}$, the first two sums of this part run over all $\langle Q, D\rangle$ with $D \mid Q$ and $|A| \geq|D|^{\delta_{1}}$, where $\delta_{1}$ will be chosen later. The last sum runs over $\bar{Q}^{\prime}$ and our goal is to count the number of $\bar{Q}^{\prime}$ such that 15 has solutions in $P, P^{\prime}$ (whose number will then be bounded by $|D|$ ). First, we consider $\bar{Q}^{\prime}$ of the form $\bar{Q}^{\prime}=C_{1}+C_{2}$, where $C_{1}$ is fixed and $C_{2}$ is an arbitrary polynomial with $\operatorname{deg} C_{2}<\operatorname{deg} A$. Plugging this into (15) and doing some simplifications yields

$$
\left|g C_{2}+L+\bar{g}\right|<|D|^{-1+\delta},
$$

where $\bar{g} \in \mathbb{L}$ does not depend on $C_{2} \in \mathbb{F}_{q}[T]$ and $L$ might depend on $C_{2}$. From the ultra-metric property of the norm, we obtain

$$
\left|\frac{B}{A} C_{2}+L+\bar{g}\right| \leq \max \left\{\left|g C_{2}+L+\bar{g}\right|,\left|R C_{2}\right|\right\}<\max \left\{|D|^{-1+\delta},|R A|\right\} .
$$

Observe that since $C_{2}$ runs through a complete set of residues modulo $A$ and $(A, B)=1, B C_{2}$ also runs through a complete set of residues modulo $A$. 
Consequently,

$$
\left|\frac{C}{A}+\bar{L}+\bar{g}\right|<\max \left\{|D|^{-1+\delta},|R A|\right\},
$$

where we now have to count the number of $C$ 's satisfying this inequality with $\operatorname{deg} C<\operatorname{deg} A$. Here, $\bar{L}$ is another polynomial that might depend on $C$. However, since the right hand side of the above inequality is smaller than 1 , $\bar{L}$ must be equal to 0 . Thus,

$$
|C+A \bar{g}|<\max \left\{|A||D|^{-1+\delta},\left|R A^{2}\right|\right\} \leq \max \left\{|A||D|^{-1+\delta}, 1\right\}
$$

and the number of such $C^{\text {'s }}$ is clearly bounded by $|A||D|^{-1+\delta}+1$. Next, observe that the number of $C_{1}$ 's above is bounded by $|Q||D A|^{-1}+1$. Therefore, the number of $\bar{Q}^{\prime}$ such that (15) has a solution in $P, P^{\prime}$ is bounded by

$$
\begin{aligned}
& \left(|A||D|^{-1+\delta}+1\right)\left(|Q||D A|^{-1}+1\right) \\
& \quad \leq|Q||D|^{-2+\delta}+|Q||D|^{-1-\delta_{1}}+\sqrt{|Q|}|D|^{-1+\delta}+1 \ll|Q||D|^{-1-\delta_{1}}+1,
\end{aligned}
$$

where $\delta_{1}, \delta$ are chosen such that $\delta+\delta_{1} \leq 1 / 2$. Overall, this yields the following bound for $\Sigma_{0}^{\prime}$ :

$$
\begin{aligned}
\Sigma_{0}^{\prime} & \ll \sum_{M \leq n \leq N} \frac{1}{q^{2 n+l_{n}}} \sum_{\substack{\operatorname{deg} Q=n \\
Q \text { monic }}} \sum_{\substack{D \mid Q \\
D \text { monic }}}\left(\frac{q^{n}}{|D|^{1+\delta_{1}}}+1\right)|D| \\
& \ll \sum_{M \leq n \leq N} \frac{1}{q^{l_{n}}}+\sum_{M \leq n \leq N} \frac{1}{q^{n+l_{n}}} \sum_{\substack{\operatorname{deg} D \leq n \\
D \text { monic }}} 1 \ll \sum_{M \leq n \leq N} \frac{1}{q^{l_{n}}} .
\end{aligned}
$$

Next, we turn to $\Sigma_{0}^{\prime \prime}$ whose first two sums run over all pairs $\langle Q, D\rangle$ with $\left.D|Q| A,|<| D\right|^{\delta_{1}}$, and $|R| \geq|D| /|Q A|$. Again, we will estimate the number of solutions of 15 in $\bar{Q}^{\prime}, P, P^{\prime}$. Therefore, first observe that 15 can be rewritten as

$$
\left|R C+\frac{L}{A}\right|<|D|^{-1+\delta}
$$

for some polynomials $C$ and $L$. If $L$ is fixed, then the number of solutions in $C$ of the above inequality is bounded by $|R|^{-1}|D|^{-1+\delta}+1$. On the other hand, we have

$$
|L| \leq \max \left\{|A||D|^{-1+\delta},|R C A|\right\} \leq \max \left\{|A||D|^{-1+\delta},|R Q A| /|D|\right\} .
$$

So, overall, for the number of $C$ 's such that there exist $L$ satisfying (17) we obtain the bound

$$
\begin{aligned}
\left(|R|^{-1}|D|^{-1+\delta}+1\right) & \left(|A||D|^{-1+\delta}+|R Q A| /|D|+1\right) \\
& \ll\left|Q A^{2}\right||D|^{-3+2 \delta}+|Q A||D|^{-2+\delta}+\sqrt{|Q|}|D|^{-1}+1 \\
& \ll|Q||D|^{-2+\delta+\delta_{1}}+\sqrt{|Q|}|D|^{-1}+1 .
\end{aligned}
$$


Note that the above number also equals the number of $\bar{Q}^{\prime}$ 's such that 14 has solutions in $P, P^{\prime}$. Hence, $\Sigma_{0}^{\prime \prime}$ is bounded as follows:

$$
\begin{aligned}
\Sigma_{0}^{\prime \prime} & \ll \sum_{M \leq n \leq N} \frac{1}{q^{2 n+l_{n}}} \sum_{\substack{\operatorname{deg} Q=n \\
Q \text { monic }}} \sum_{\substack{D \mid Q \\
D \text { monic }}}\left(\frac{q^{n}}{|D|^{2-\delta-\delta_{1}}}+\frac{q^{n / 2}}{|D|}+1\right)|D| \\
& \ll \sum_{M \leq n \leq N} \frac{1}{q^{l_{n}}}+\sum_{M \leq n \leq N} \frac{1}{q^{n / 2+l_{n}}} \sum_{\substack{\operatorname{deg} D \leq n \\
D \text { monic }}} \frac{1}{|D|} \\
& \ll \sum_{M \leq n \leq N} \frac{1}{q^{l_{n}}}+\sum_{M \leq n \leq N} \frac{n}{q^{n / 2+l_{n}}} \ll \sum_{M \leq n \leq N} \frac{1}{q^{l_{n}}} .
\end{aligned}
$$

So, what is left is to bound $\Sigma_{0}^{\prime \prime \prime}$. Here, the first two sums run over all pairs $\langle Q, D\rangle$ with $\left.D|Q| A,|<| D\right|^{\delta_{1}}$, and $|R|<|D| /|Q A|$. Then (15) together with the ultra-metric property of the norm yields

$$
\begin{aligned}
& \left|\bar{Q}\left(A P^{\prime}+B\right)-\bar{Q}^{\prime}(A P+B)\right| \\
& \leq \max \left\{\left|R\left(\bar{Q}-\bar{Q}^{\prime}\right) A\right|,|A|\left|g\left(\bar{Q}-\bar{Q}^{\prime}\right)+P^{\prime} \bar{Q}-P \bar{Q}^{\prime}\right|\right\}<1 .
\end{aligned}
$$

Consequently,

$$
\bar{Q}\left(A P^{\prime}+B\right)=\bar{Q}^{\prime}(A P+B) .
$$

Thus $A P+B \equiv 0 \bmod \bar{Q}$ and this implies $\operatorname{deg} \bar{Q} \leq \Gamma(n)$. The latter in turn yields $\operatorname{deg} D \geq n-\Gamma(n)$. So, in this case, we obtain the bound

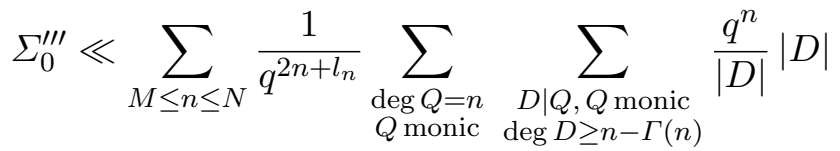

$$
\begin{aligned}
& =\sum_{M \leq n \leq N} \frac{1}{q^{n+l_{n}}} \sum_{\substack{\operatorname{deg} Q=n \\
Q \text { monic }}} \sum_{\substack{\operatorname{deg} D \\
\operatorname{deg}(n)}} 1 \ll \sum_{M \leq n \leq N} \frac{\Gamma(n)}{q^{l_{n}}} .
\end{aligned}
$$

Finally, combining (16), (18), and (19) gives the bound

$$
\Sigma_{0} \ll \sum_{M \leq n \leq N} \frac{\Gamma(n)}{q^{l_{n}}} .
$$

Plugging this into (14) then proves the claimed result.

Now, we can start with the proof of Theorem 1 .

Proof of Theorem 1. First, from Proposition 4 together with Lemma 2 , we obtain

$$
\sum_{n \leq N} \xi_{n}^{*}=\Psi(N)+\mathcal{O}\left(\Psi^{*}(N)^{1 / 2}\left(\log \Psi^{*}(N)\right)^{3 / 2+\epsilon}\right), \quad \text { a.s. }
$$


where $\epsilon>0$ is an arbitrary constant. Next, observe

$$
\Psi^{*}(N)=\sum_{n \leq N} \frac{\Gamma(n)}{q^{l_{n}}} \ll \Psi(N) \log \Psi(N) .
$$

Hence, the claimed result holds for the sequence $\xi_{n}^{*}$.

In order to show that it holds for $\xi_{n}$ as well, observe that from Proposition 3 ,

$$
P\left(\sum_{n \leq N}\left(\xi_{n}-\xi_{n}^{*}\right)>\log \Psi(N)\right) \ll(\log \Psi(N))^{-1} .
$$

Next, choose $N_{k}$ to be the minimal positive integer with $\log \Psi\left(N_{k}\right) \geq 2^{k}$. Then, the Borel-Cantelli lemma implies that

$$
\sum_{n \leq N_{k}}\left(\xi_{n}-\xi_{n}^{*}\right) \leq \log \Psi\left(N_{k}\right)
$$

for almost all $f$ and $k$ large enough. Now, let $N$ be a large enough integer with $N_{k} \leq N<N_{k+1}$. Then

$$
\sum_{n \leq N}\left(\xi_{n}-\xi_{n}^{*}\right) \leq \sum_{n \leq N_{k+1}}\left(\xi_{n}-\xi_{n}^{*}\right) \leq \log \Psi\left(N_{k+1}\right) \ll \log \Psi\left(N_{k}\right) \ll \log \Psi(N) .
$$

Overall, we have shown that for almost all $f$,

$$
\sum_{n \leq N} \xi_{n}=\sum_{n \leq N} \xi_{n}^{*}+\mathcal{O}(\log \Psi(N)) .
$$

Combining this with the above result yields the claim.

We note that Theorem 2 also follows from the method above with only minor modifications. So, what is left is the proof of Corollary 1 .

Proof of Corollary 1 . For (i), choose $F$ such that

$$
\mathcal{F}=\left\{C+L D: C+L D \text { monic and } L \in \mathbb{F}_{q}[T]\right\} .
$$

Then $\# \mathcal{F}_{n}=q^{n} /|D|$ for all $n \geq \operatorname{deg} D$. Consequently,

$$
\Psi(N, \mathcal{F})=\frac{1}{|D|} \Psi(N)+\mathcal{O}(1) .
$$

For (ii), it suffices to point out that it is well-known (see Chapter 3 in [1) that the number of monic, square-free polynomials of degree $n \geq 2$ is $q^{n}-q^{n-1}$. Hence,

$$
\Psi(N, \mathcal{F})=\frac{q-1}{q} \Psi(N)+\mathcal{O}(1) .
$$

From this the result follows. 
4. The "double-metric" and the other "single-metric" case. We first turn our attention to the "double-metric" case. So, in the following, we consider (1) with both $f, g$ random. As before, we define the set

$F_{Q}:=\left\{\langle f, g\rangle \in \mathbb{L} \times \mathbb{L}:\langle f, g\rangle\right.$ is a solution of (1) with some $\left.P \in \mathbb{F}_{q}[T]\right\}$, where $Q$ is a non-zero polynomial.

As already mentioned in the introduction, this case is much easier than the "single-metric" case discussed in the previous sections. The reason for this is the second property of the following lemma which was proved in [8].

LEMMA 5.

$$
(m \times m)\left(F_{Q}\right)=\frac{1}{q^{n+l_{n}}},
$$

$$
(m \times m)\left(F_{Q} \cap F_{Q^{\prime}}\right)=(m \times m)\left(F_{Q}\right)(m \times m)\left(F_{Q^{\prime}}\right) \quad \text { for } Q \neq Q^{\prime} .
$$

So, if we define

$$
\xi_{n}:=\#\{\langle P, Q\rangle:\langle P, Q\rangle \text { is a solution of (1) }\},
$$

then we again have

$$
\xi_{n}=\sum_{\substack{\operatorname{deg} Q=n \\ Q \text { monic }}} \mathbf{1}_{F_{Q}} .
$$

However, the above lemma shows that $\xi_{n}$ considered as a sequence of random variables on the product probability space is pairwise independent. This yields

$$
\begin{aligned}
\mathbb{E}\left(\sum_{M \leq n \leq N} \xi_{n}-\frac{1}{q^{l_{n}}}\right)^{2} & =\sum_{M \leq n \leq N} \operatorname{Var}\left(\xi_{n}\right)=\sum_{M \leq n \leq N} \frac{1}{q^{l_{n}}}\left(1-\frac{1}{q^{n+l_{n}}}\right) \\
& =\sum_{M \leq n \leq N} \frac{1}{q^{l_{n}}}+\mathcal{O}(1) .
\end{aligned}
$$

Hence, if we assume that

$$
\Psi(N):=\sum_{n \leq N} \frac{1}{q^{l_{n}}} \rightarrow \infty \quad \text { as } N \rightarrow \infty,
$$

then Lemma 2 directly applies and yields the following result (whose proof in case the above assumption does not hold is trivial).

Theorem 4. The number of solutions of (1) with $0 \leq \operatorname{deg} Q \leq N$ is

$$
\Psi(N)+\mathcal{O}\left(\Psi(N)^{1 / 2}(\log \Psi(N))^{3 / 2+\epsilon}\right), \quad \text { a.s. },
$$

where $\epsilon>0$ is an arbitrary constant.

Note that a.s. here means with respect to the product measure $m \times m$.

Finally, we briefly discuss the other "single-metric" case where the roles of $f$ and $g$ are interchanged. Therefore, assume now that $f$ is fixed and $g$ is random. Here, without proof, we state the following result: for any sequence 
$l_{n}$ tending to infinity arbitrarily slowly, there exists an $f \in \mathbb{L}$ such that for almost all $g$ the number of solutions of (1) is finite (see P. Szüsz [12] for the corresponding result in the real number case). Consequently, results similar to those above are impossible in this case.

Acknowledgments. Parts of this work were carried out when the author visited the Department of Mathematics, Keio University. The author wants to thank the department for hospitality and support. Moreover, the author acknowledges partial support by National Science Council under the grant NSC-98-2115-M-009-009.

\section{References}

[1] E. R. Berlekamp, Algebraic Coding Theory, McGraw-Hill, 1968.

[2] V. Berthé, H. Nakada, and R. Natsui, Asymptotic behavior of the number of solutions for non-Archimedean Diophantine approximations with restricted denominators, Finite Fields Appl. 14 (2008), 849-866.

[3] J. W. S. Cassels, An Introduction to Diophantine Approximation, Cambridge Univ. Press, 1951.

[4] M. M. Dodson, S. Kristensen, and J. Levesley, A quantitative Khintchine-Groshev type theorem over a field of formal series, Indag. Math. 16 (2005), 171-177.

[5] M. Drmota and D. Panario, A rigorous proof of the Waterloo algorithm for the discrete logarithm problem, Des. Codes Cryptogr. 26 (2002), 229-241.

[6] M. Fuchs, An analogue of a theorem of Szüsz for formal Laurent series over finite fields, J. Number Theory 101 (2003), 105-130.

[7] G. Harman, Metric Number Theory, London Math. Soc. Monogr. (N.S.) 18, Oxford Univ. Press, New York, 1998.

[8] C. Ma and W.-Y. Su, Inhomogeneous Diophantine approximation over the field of formal Laurent series, Finite Fields Appl. 14 (2008), 361-378.

[9] H. Nakada and R. Natsui, Asymptotic behavior of the number of solutions for nonArchimedean Diophantine approximations, Acta Arith. 125 (2006), 203-214.

[10] W. M. Schmidt, A metrical theorem in Diophantine approximation, Canad. J. Math. 12 (1960), 619-631.

[11] -, Metrical theorems on fractional parts of sequences, Trans. Amer. Math. Soc. 110 (1964), 493-518.

[12] P. Szüsz, Über die metrische Theorie der Diophantischen Approximation, Acta Math. Hungar. 9 (1958), 177-193.

Michael Fuchs

Department of Applied Mathematics

National Chiao Tung University

1001 Ta Hsue Road

Hsinchu, 300, Taiwan

E-mail: mfuchs@math.nctu.edu.tw 